\title{
S-mixing and quantum tunneling of the magnetization in molecular nanomagnets
}

\author{
S. Carretta, E. Liviotti, N. Magnani, P. Santini and G. Amoretti \\ Istituto Nazionale per la Fisica della Materia, \\ Dipartimento di Fisica, Università di Parma, I-43100 Parma, Italy
}

(Dated: January 21, 2004)

\begin{abstract}
The role of $S$-mixing in the quantum tunneling of the magnetization in nanomagnets has been investigated. We show that the effect on the tunneling frequency is huge and that the discrepancy (more than 3 orders of magnitude in the tunneling frequency) between spectroscopic and relaxation measurements in $\mathrm{Fe}_{8}$ can be resolved if $S$-mixing is taken into account.
\end{abstract}

PACS numbers: 75.45.+j,82.20.Xr,75.30.Gw 
Molecular nanomagnets (MNMs) [1, 2] are molecules containing transition-metal ions whose spins are so strongly exchange-coupled that at low temperature each molecule behaves like a single-domain particle with fixed total spin. One of the most interesting phenomena displayed by these systems is quantum tunneling of the direction of the total spin through energy barriers [3, 4, 5]. The measured step-like magnetization curves of $\mathrm{Mn}_{12}$ and $\mathrm{Fe}_{8}$ provided macroscopic evidence of relaxation through quantum tunneling. The latter is revealed by resonances observed in the relaxation rate at specific values of the external magnetic field $B_{A C}$, at which energy levels on opposite sides of the anisotropy barrier are nearly degenerate and anticrossings (ACs) in the field dependence of the energies occur. The relaxation rate depends crucially on the value of the so-called tunnel splitting $\Delta$, i.e. the gap at $B_{A C}$ between the quasi-degenerate states. In particular, at very low temperature $T$ and at short times $t$ the magnetization relaxes as $1-\sqrt{\Gamma t}$ where $\Gamma \propto \Delta^{2}[6]$. For $\mathrm{Fe}_{8}, \Delta$ was extracted in Ref. [5] by measuring with a microsquid apparatus the magnetization steps induced by sweeping a longitudinal (i.e. parallel to the easy axis) applied field $B_{z}$ across $B_{A C}$. The size of these steps was linked to the tunnel splitting through the Landau-Zener formula

$$
\Pi=1-e^{-\Delta^{2} / A}
$$

where $\Pi$ is the tunneling probability at a level anticrossing, and $A$ is proportional to the field sweeping rate. When the experiment is performed in a static transverse field $B_{y}, \Delta$ is found to display oscillations as a function of $B_{y}$, which in a semiclassical approach reflect the destructive interference of tunneling pathways.

Eq. (1) had been deduced by neglecting decoherence sources such as hyperfine and dipolar fields. Nevertheless, it remains valid if the sweeping rate is as fast as that actually used in the experiments[7]. In addition, the model proposed in Ref. [8] shows that the incoherent Zener tunneling can be described by Eq. (1) with $\Delta$ renormalized by a factor $\sqrt{2}$.

A striking circumstance is that the measured value of $\Delta$ seems completely incompatible with the value calculated by using the Hamiltonian determined by inelastic neutron scattering (INS) 9, 10], optical spectroscopy [11] and electron paramagnetic resonance 12]. Indeed, the measured zero-field gap $\Delta\left(B_{y}=0\right)$ between the two lowest levels is near $10^{-7} \mathrm{~K}[\underline{5}]$, while the value calculated $\left(4.44 \cdot 10^{-11} \mathrm{~K}\right)$ is more than 3 orders of magnitude smaller. This huge discrepancy seriously hinders any attempt to reach a satisfactory theoretical modelling of the quantum tunneling of the magnetization, where the square of $\Delta$ plays a fundamental 
role. The purpose of this work is to show that commonly neglected quantum fluctuations of the magnitude of the total spin of the molecule (S-mixing [13, 14]) affect the tunnel splitting of $\mathrm{Fe}_{8}$ hugely, and allow the above-mentioned discrepancy to be solved. Since $\mathrm{Fe}_{8}$ displays a relatively small degree of $S$-mixing, we expect the tunnel splitting of many nanomagnets to be influenced even more heavily than in $\mathrm{Fe}_{8}$ by such fluctuations.

Each $\mathrm{Fe}_{8}$ molecule can be described by the following spin Hamiltonian:

$$
\begin{gathered}
H=\sum_{i>j} J_{i j} \mathbf{s}_{i} \cdot \mathbf{s}_{j}+\sum_{i} \sum_{k, q} b_{k}^{q}(i) O_{k}^{q}\left(\mathbf{s}_{\mathbf{i}}\right)+ \\
\sum_{i>j} \mathbf{s}_{i} \cdot \mathbf{D}_{i j} \cdot \mathbf{s}_{j}-g \mu_{B} \sum_{i} \mathbf{B} \cdot \mathbf{s}_{i},
\end{gathered}
$$

where $\mathbf{s}_{i}$ are spin operators of the $i^{t h} \mathrm{Fe}^{3+}$ ion in the molecule $\left(s_{i}=5 / 2\right)$. The first term is the isotropic Heisenberg exchange interaction. The second term describes the local crystalfields (CFs), with $O_{k}^{q}\left(\mathbf{s}_{\mathbf{i}}\right)$ Stevens operator equivalents for the $i$-th ion [16] and $b_{k}^{q}(i) \mathrm{CF}$ parameters. Here $k=2$ or 4 (larger values of the rank $k$ are forbidden for $d$-electrons [16]), and $q=-k, \ldots, k$. The third term represents the dipolar anisotropic intra-cluster spin-spin interactions. The last term is the Zeeman coupling with an external field $\mathbf{B}$. The exchange constants $J_{i j}$ used in this work are those determined from susceptibility [17].

While the Heisenberg term is rotationally invariant and therefore conserves the length $|\mathbf{S}|$ of the total spin $\mathbf{S}=\sum_{i} \mathbf{s}_{i}$, the anisotropic terms do not conserve this observable. Nevertheless, since the Heisenberg contribution is usually largely dominant, $|\mathbf{S}|$ is nearly conserved, and the energy spectrum of $H$ consists of a series of level multiplets with an almost definite value of $|\mathbf{S}|$. By neglecting the mixing between different $S$-multiplets (i.e. $S$ mixing), the Hamiltonian Eq. (2) can be projected onto each $S$-multiplet (strong-exchange limit):

$$
\begin{gathered}
H_{\text {sub }}=B_{2}^{0} O_{2}^{0}(\mathbf{S})+B_{2}^{2} O_{2}^{2}(\mathbf{S})+B_{4}^{0} O_{4}^{0}(\mathbf{S}) \\
+B_{4}^{2} O_{4}^{2}(\mathbf{S})+B_{4}^{4} O_{4}^{4}(\mathbf{S})-g \mu_{B} \mathbf{B} \cdot \mathbf{S}
\end{gathered}
$$

where $\mathbf{S}$ is a vector spin operator with $S$ equal to the total spin of the $S$-multiplet [18]. The parameters $B_{K}^{Q}$ are calculated from $b_{k}^{q}(i)$ and $\mathbf{D}_{i j}$ by $\mathrm{CF}$ and dipolar projection coefficients. This approach, applied to the $S=10$ ground manifold of $\mathrm{Fe}_{8}$, has allowed to interpret INS data very satisfactorily by assuming $B_{2}^{0}=-9.75 \times 10^{-2} \mathrm{~K}, B_{2}^{2}=-4.66 \times 10^{-2} \mathrm{~K}, B_{4}^{0}=$ $1.0 \times 10^{-6} \mathrm{~K}, B_{4}^{2}=1.2 \times 10^{-7} \mathrm{~K}, B_{4}^{4}=8.6 \times 10^{-6} \mathrm{~K}[\mathrm{9}, 10]$. Very similar parameter values 
are obtained from optical spectroscopy [11] and electron paramagnetic resonance[12]. In particular $B_{4}^{4}$, which has the greatest effect on $\Delta$, is the same. In order to reproduce the measured magnitude and oscillations of $\Delta$ with Eq. (3), values of $B_{K}^{Q}$ incompatible with neutron results had to be assumed [5, 8, 19]. In particular, $B_{4}^{4}$ was one order of magnitude larger and its sign was reversed. In Fig. 1 we show calculations at $T=9.6 K$ of the INS spectrum of $\mathrm{Fe}_{8}$ powder with the experimental resolution of Ref. [9]. The parameters used in Refs. [5, 8, 19] do not reproduce the INS spectra satisfactorily, neither in the higher-energy part measured in Ref. [9] nor in the lower-energy part measured with the high resolution experiment of Ref.[10]. If the strong-exchange-limit Hamiltonian (3) is used, there is no way to reproduce with a unique set of parameters the magnitude and behavior of $\Delta$ and the spectroscopic results. In the following we show how this discrepancy can be removed if $S$-mixing is taken into account. Indeed, although in $\mathrm{Fe}_{8} S$-mixing is a small perturbation (e.g., it produces negligible changes in calculated spectroscopic quantities), its effect on $\Delta$ is very large because it provides much more efficient tunneling channels.

In order to evaluate $S$-mixing effects, we followed the method developed in [13], in which $S$-mixing is included up to the second order in the anisotropy by a unitary transformation applied to the complete Hamiltonian (2). The system can be still described as an effective spin $S=10$, provided the spin-Hamiltonian (3) is properly modified : the parameters of the Stevens operators are renormalized, and new higher rank $(K>4)$ terms are added. These latter are forbidden for $d$-electrons in the strong-exchange limit. The advantage of using this method with respect to large-scale numerical diagonalization (e.g. using the Lanczos algorithm) is twofold: first of all, it allows calculation times to be reduced drastically. In fact, the time-consuming part of calculation (i.e. computing $\Xi$ and $\Upsilon$ in Eq. (9)) does not depend on the specific set of local CF parameters, and therefore has to be performed only once. The second advantage is that the simple and physically transparent single-spin formalism of the strong-exchange limit is recovered.

Using as basis vectors the eigenvectors $|\alpha S M\rangle$ of the isotropic exchange $H_{0}$, the full Hamiltonian matrix $H$ Eq. (2) can be written as the sum of three terms

$$
H=H_{0}+H_{1}+H_{2}
$$

where $H_{1}+H_{2}$ represents the anisotropic interactions. $H_{1}$ has nonzero elements only within the $S$ multiplets, while $\mathrm{H}_{2}$ joins states with different $\alpha S$ and is the term responsible for the 
mixing. $H_{2}$ is neglected in the strong-exchange limit Eq. (3).

The perturbational procedure [13, 15] consists in performing a unitary transformation on $H$ such that the off-diagonal (in $\alpha S$ ) blocks of the transformed Hamiltonian $H^{\prime}$ are zero up to second order in the anisotropy. Hence, in the new basis, states belonging to different multiplets are uncoupled and the system can be described as an effective spin multiplet, like in the strong-exchange limit. The matrix elements of $H^{\prime}$ inside the ground multiplet $S=10$ are given by

$$
\begin{gathered}
\left\langle S M\left|H^{\prime}\right| S M^{\prime}\right\rangle=E_{0} \delta_{M, M^{\prime}}+\left\langle\alpha S M\left|H_{1}\right| \alpha S M^{\prime}\right\rangle \\
-\sum_{\alpha^{\prime \prime} S^{\prime \prime} M^{\prime \prime}} \frac{\left\langle\alpha S M\left|H_{2}\right| \alpha^{\prime \prime} S^{\prime \prime} M^{\prime \prime}\right\rangle\left\langle\alpha^{\prime \prime} S^{\prime \prime} M^{\prime \prime}\left|H_{2}\right| \alpha S M^{\prime}\right\rangle}{E_{0 \alpha^{\prime \prime} S^{\prime \prime}}-E_{0}}
\end{gathered}
$$

where $E_{0}$ is the lowest eigenvalue of $H_{0}$ and $|\alpha S M\rangle$ are the corresponding eigenvectors. $\left|\alpha^{\prime \prime} S^{\prime \prime} M^{\prime \prime}\right\rangle$ are excited eigenvectors of $H_{0}$ with energy $E_{0 \alpha^{\prime \prime} S^{\prime \prime}}$.

The second term in Eq. (5) coincides with the strong-exchange Hamiltonian (3), while the last term represents mixing corrections. By exploiting the Wigner-Eckart theorem[13] the latter can be written in general as

$$
\sum_{K, Q} \tilde{B}_{K}^{Q} O_{K}^{Q}
$$

with $K \leq 8$ and even, and $-K \leq Q \leq K$. Hence

$$
H_{1}+H_{2}=\sum_{K, Q} C_{K}^{Q} O_{K}^{Q}
$$

where $C_{K}^{Q}=B_{K}^{Q}+\tilde{B}_{K}^{Q}\left(B_{K}^{Q}=0\right.$ for $\left.K>4\right)$. Therefore, on the one hand $S$-mixing introduces in the effective Hamiltonian new terms forbidden in the strong-exchange limit, and on the other hand it renormalizes the coefficients of the other terms (with $K \leq 4$ ). This implies that the CF parameters determined by INS are to be regarded as $C_{K}^{Q}$ s rather than as the $B_{K}^{Q} \mathrm{~S}$ of Eq. (3).

The parameters $\tilde{B}_{K}^{Q}$ are given by linear combinations of products of reduced matrix elements. For example,

$$
\tilde{B}_{6}^{6}=\sum_{i, j} b_{2}^{2}(i) b_{4}^{4}(j) \Xi(i, j)+\sum_{i} b_{4}^{4}(i) \Upsilon(i)
$$

with

$$
\Xi(i, j)=\sum_{S^{\prime \prime}=S-2}^{S+2} c_{66}^{S-S^{\prime \prime}} .
$$




$$
\sum_{\alpha^{\prime \prime}} \frac{\left\langle\alpha S\left\|T^{(2)}(i)\right\| \alpha^{\prime \prime} S^{\prime \prime}\right\rangle\left\langle\alpha S\left\|T^{(4)}(j)\right\| \alpha^{\prime \prime} S^{\prime \prime}\right\rangle}{E_{0 \alpha^{\prime \prime} S^{\prime \prime}}-E_{0}}
$$

and

$$
\begin{gathered}
\Upsilon(i)=-\sqrt{2} \sum_{j, k} J_{j k}^{u} \sum_{S^{\prime \prime}=S-2}^{S+2} c_{66}^{S-S^{\prime \prime}} . \\
\sum_{\alpha^{\prime \prime}} \frac{\left\langle\alpha S\left\|T^{(2)}(11 \mid j k)\right\| \alpha^{\prime \prime} S^{\prime \prime}\right\rangle\left\langle\alpha S\left\|T^{(4)}(i)\right\| \alpha^{\prime \prime} S^{\prime \prime}\right\rangle}{E_{0 \alpha^{\prime \prime} S^{\prime \prime}}-E_{0}} .
\end{gathered}
$$

$i, j$ and $k$ label magnetic ions, $T^{(K)}(i)$ and $T^{(2)}(11 \mid j k)$ are the tensor operators describing the local CF and dipole-dipole interactions [20]. $J_{j k}^{u}$ are defined in terms of the elements of $\mathbf{D}_{i j}$ (Eq. (2)) in Ref. 20]. The $c_{66}^{S-S^{\prime \prime}}$ coefficients are defined according to the theory developed in [13]. Expressions similar to (8), (9a) and (9b) are obtained for the other parameters $\tilde{B}_{K}^{Q}$, which are all expressed as polynomials of $2^{n d}$-order in the $b_{k}^{q}(i)$. Hence $S$-mixing gives rise to highly efficient and otherwise forbidden tunneling channels, by generating new high-rank anisotropy terms.

In order to assess the impact of these terms on the tunnel-splitting we applied our theory quantitatively. While the dipole-dipole interaction (appearing, e.g., in (9b) through $J_{j k}^{u}$ ) can be computed by the point-dipole approximation[21], the local CF parameters $b_{k}^{q}(i)$ cannot be determined $a b$ initio reliably. Therefore, by numerically inverting the $2^{\text {nd }}$-order functions $C_{K}^{Q}\left(\left\{b_{k}^{q}(i)\right\}\right)$, we determine the possible sets $\left\{b_{k}^{q}(i)\right\}_{f}$ consistent with INS, i.e. such that the values $C_{K}^{Q}\left(\left\{b_{k}^{q}(i)\right\}_{f}\right)$ for $K \leq 4$ coincide with those determined by INS (within experimental error bars). Even by neglecting all $b_{k}^{q}(i)$ with $q \neq 0,2,4$ (i.e. those not contributing to $H_{1}$ ) and by enforcing on the $\left\{b_{k}^{q}(i)\right\}$ the approximate $D_{2}$ molecular symmetry of $\mathrm{Fe}_{8}[22]$, there are still more unknown parameters than constraints, and we find therefore that there are infinitely many sets compatible with INS. For these sets, the distribution of the calculated values of the tunnel splitting $\Delta$ is shown in Fig. 2a by a histogram of the log-increments $\log \left(\Delta / \Delta_{0}\right)$. Here the local CF parameters $b_{k}^{q}(i)$ vary on a grid bounded by $\left|b_{2}^{q}(i)\right|<8 \mathrm{~K}$, $\left|b_{4}^{q}(i)\right|<0.4 \mathrm{~K}$. This choice is based on two considerations: $i$ ) the experimental values of $C_{2}^{0}$ and $C_{2}^{2}$ set only a lower bound $\left|b_{2}^{q}(i)\right| \gtrsim 1 K$. Local second-order parameters of the order of few $K$ are reasonable in case of $F e^{3+}$ in a low symmetry environment [23]. ii) Typical ratios of fourth- to second-order CF parameters range (in modulus) between 0.01 and 0.1. Fig. 2a shows that $S$-mixing plays a crucial role since typically it enhances $\Delta$ by several orders of magnitude. Fig. 2b reports the result of the same calculation when the grid bounds are restricted to $\left|b_{2}^{q}(i)\right|<4 \mathrm{~K},\left|b_{4}^{q}(i)\right|<0.04 \mathrm{~K}$. Even with this more restrictive choice the effect 
of $S$-mixing remains huge. The measured value of $\Delta$ is indicated by an arrow and falls well inside the distribution.

We have shown that $S$-mixing can remove the discrepancy between the value of the zerofield gap $\Delta\left(B_{z}=0, B_{y}=0\right)$ measured by relaxation experiments and that calculated from the spectroscopic results. Now it remains to prove that also the measured oscillations of $\Delta\left(B_{z}=0, B_{y}\right)$, and the behavior of the AC gaps $\Delta_{e x}\left(B_{z}=B_{A C}, B_{y}\right)$ between excited states are reproducible. The aim of this work is not to perform a best-fit of the observed oscillations of the tunnel-splittings, but to prove that $S$-mixing eliminates the inconsistency between spectroscopic and Landau-Zener measurements. Therefore, we limit Eq.(6) to values of $K \leq 6$ in order to find among the infinite possible parameter sets consistent with INS, one involving as few high-rank terms as possible, and reproducing the AC gaps behavior satisfactorily. The new terms in Eq.(7) (forbidden in the strong-exchange limit) with significative influence on $\Delta$ are then $C_{6}^{4}$ and $C_{6}^{6}$.

As a first step, we fixed $C_{K}^{Q}$ for $K \leq 4$ to the values determined by neutron spectroscopy (reported below Eq. (3)) and we chose values of $C_{6}^{4}$ and $C_{6}^{6}$ which reproduce the behavior of $\Delta$ satisfactorily. With $C_{6}^{4} \sim-1.8 \times 10^{-7} \mathrm{~K}$ and $C_{6}^{6} \sim-1.15 \times 10^{-7} \mathrm{~K}, \Delta$ calculated at zero applied field is $\sim 1.1 \times 10^{-7} \mathrm{~K}$, to be compared with the value $\sim 0.4 \times 10^{-10} \mathrm{~K}$ obtained when $C_{6}^{4}=0$ and $C_{6}^{6}=0$. Moreover, the measured oscillations of $\Delta$ as a function of the transverse field $B_{y}$ are well reproduced, as well as the $\mathrm{AC}$ gaps involving the excited states $|-10\rangle$ and $|10-n\rangle(n=1,2)$ (see Fig. 3). The nonzero value of experimental oscillations at minima may arise from experimental artifacts, (e.g., crystal mosaicity). The behavior of $\Delta$ as a function of the transverse field modulus for nonzero azimuthal angles $\phi$ between the applied field and the $y$-axis, is also in good agreement with measurements [5] .

As a second step, we checked that the addition of these 6th-order terms does not affect the INS cross-section significantly. In fact, the recalculated cross-section is indistinguishable from that calculated in [9] and reported in Fig.1, apart from an irrelevant shift (by $\sim 20$ $\mu \mathrm{eV}$ ) of the shoulder at $0.16 \mathrm{meV}$.

Values of $C_{6}^{4}$ and $C_{6}^{6}$ of the order and sign of those given above are realistic in $\mathrm{Fe}_{8}$. For example, the insets in Figs. $2 \mathrm{a}$ and $2 \mathrm{~b}$ show the distribution $P\left(C_{6}^{6}\right)$ of values of $C_{6}^{6}$ calculated on the same grids as described above. We stress that our choice of high-rank parameters is merely the simplest possibility. There are many different sets involving also the other high-rank terms which would be consistent with experimental data. Although a 
unique determination of the $C_{K}^{Q} \mathrm{~s}$ is not possible, the important point is that the addition of high-rank terms, which is allowed only if $S$-mixing is considered, is essential to describe consistently relaxation and spectroscopic data.

In conclusion, we have shown that the discrepancy (more than 3 orders of magnitude in the tunnel splitting) between spectroscopy and relaxation measurements in $\mathrm{Fe}_{8}$ can be resolved if $S$-mixing is taken into account. Even a small degree of $S$-mixing has huge influence in the tunneling dynamics since it opens highly efficient tunnel channels through otherwise forbidden high-rank anisotropy terms. The degree of $S$-mixing is strongly influenced by the topology of the molecule. Therefore in addition to the height of the anisotropy barrier, also the cluster topology must be taken into account in designing new nanomagnets.

[1] R. Sessoli et al., Nature 365, 141 (1993).

[2] D. Gatteschi et al., Science 265, 1054 (1994).

[3] J. R. Friedman et al., Phys. Rev. Lett. 76, 3830 (1996).

[4] L. Thomas et al., Nature 383, 145 (1996).

[5] W. Wernsdorfer and R. Sessoli, Science 284, 133 (1999).

[6] N.V. Prokof'ev and P.C.E. Stamp, Phys. Rev. Lett. 80, 5794 (1998).

[7] N.A. Sinitsyn and N.V. Prokof'ev, Phys. Rev. B 67, 134403 (2003).

[8] M.N. Leuenberger and D. Loss, Phys. Rev. B 61, 12200 (2000).

[9] R. Caciuffo et al., Phys. Rev. Lett. 81, 4744 (1998).

[10] G. Amoretti et al., Phys. Rev. B 62, 3022 (2000).

[11] A. Mukhin et al., Phys. Rev. B 63, 214411 (2001).

[12] S. Hill et al., Phys. Rev. B 65, 224410 (2002).

[13] E. Liviotti et al., J. Chem. Phys. 117, 3361 (2002).

[14] S. Carretta et al., Eur. Phys. J. B 36, 169 (2003).

[15] C.P. Slichter, Principles of Magnetic Resonance, Springer-Verlag (NY) (1992).

[16] A. Abragam and B. Bleaney, Electron paramagnetic resonance of transition ions, Clarendon Press, Oxford (1970).

[17] A.L. Barra et al., Chem. Eur. J. 6, 1608 (2000).

[18] Other second- and fourth-order terms are expected to be negligibly small because of the 
approximate $D_{2}$ symmetry of the molecule.

[19] E. Rastelli and A. Tassi, Phys. Rev. B 65, 092413 (2002).

[20] J.J. Borrás-Almenar et al., Inorg. Chem. 38, 6081 (1999).

[21] A. Bencini and D. Gatteschi, EPR of Exchange Coupled Systems, Springer Verlag, Berlin (1990).

$[22] b_{k}^{q}(1)=b_{k}^{q}(2), b_{k}^{q}(3)=b_{k}^{q}(4), b_{k}^{q}(5)=b_{k}^{q}(6)=b_{k}^{q}(7)=b_{k}^{q}(8), q=0,2,4$ (indices are defined in the inset of Fig. 1). To further reduce the number of free parameters we assumed $b_{4}^{4}(1)=b_{4}^{4}(3)$.

[23] See, e.g., E. Siegel and K.A. Müller, Phys. Rev. B 19, 109 (1979). 


\section{FIGURE CAPTIONS:}

Fig.1: Calculated INS intensity for a $\mathrm{Fe}_{8}$ powder with the spin Hamiltonian Eq. (3) and various parameter sets. The energy resolution has been fixed to the experimental value of $19 \mu \mathrm{eV}$ [9]. The parameters used in Ref. 19] are close to those used in Ref. [5] and yield almost the same intensity curve. The inset shows a schematic view of $\mathrm{Fe}_{8}$.

Fig.2: Calculated distribution of values of the tunnel splitting $\Delta\left(B_{z}=0, B_{y}=0\right)$ normalized to the value $\Delta_{0}=4.44 \cdot 10^{-11} \mathrm{~K}$ obtained without $S$-mixing (with the parameters obtained from INS). The local CF parameters $b_{k}^{q}(i)$ vary on grids (different in (a) and (b)) defined in the text. Arrows indicate the measured ratios. Insets show the distribution of values of $C_{6}^{6}$ on the same grids.

Fig.3: Top: measured tunnel splitting as function of an applied transverse magnetic field $B_{y}$ with $B_{z}=0(n=0)$, and $\mathrm{AC}$ gaps involving the excited states $|-10\rangle$ and $|10-n\rangle$ $(n=1,2)$ with $B_{z}=B_{A C} \sim n \cdot 0.22 \mathrm{~T}(n=1,2)[5]$. Bottom: the same quantities calculated with the Hamiltonian $(7)$ and the $C_{K}^{Q}$ parameters given in the text. 
This figure "fig1.jpg" is available in "jpg" format from: http://arxiv.org/ps/cond-mat/0404011v1 
This figure "fig2.jpg" is available in "jpg" format from: http://arxiv.org/ps/cond-mat/0404011v1 
This figure "fig3.jpg" is available in "jpg" format from: http://arxiv.org/ps/cond-mat/0404011v1 